\title{
The efficacy and safety of antimuscarinics for the prevention or treatment of catheter- related bladder discomfort: a systematic review and meta-analysis of randomized controlled trials
}

\author{
Zhongbao Zhou ${ }^{1 \dagger}$, Yuanshan Cui ${ }^{1,2+}$, Xiaoyi Zhang ${ }^{3}$, Youyi Lu², Zhipeng Chen ${ }^{4^{*}}$ and Yong Zhang ${ }^{1 *}$ (i)
}

\begin{abstract}
Objectives: This meta-analysis aimed to evaluate the efficacy and safety of antimuscarinics for the prevention or treatment of catheter related bladder discomfort (CRBD).

Methods: The MEDLINE, EMBASE, and Cochrane Controlled Trials Register (from 1987 to July 2021) were used to search randomized controlled trials. The PRISMA checklists were followed. RevMan5.4.0 was used for statistical analysis.
\end{abstract}

Results: Eleven studies involving 1165 patients were involved in the analysis. The study reported that the incidence of CRBD observed in the antimuscarinics group was significantly lower than that of the control group at 0-, 1-, 2-, and 6-h after drug therapy $(P=0.001, P<0.0001, P=0.0005$, and $P=0.001$, respectively). For side effects, there were not statistical differences between the antimuscarinics group and the control group, mainly including dry mouth (risk ratio $(\mathrm{RR})=1.31,95 \%$ confidence interval $(C \mathrm{I})=0.95$ to $1.80, P=0.09$ ), postoperative nausea and vomiting ( $R R=1.02,95 \% \mathrm{Cl}=0.55$ to $1.90, P=0.87)$, facial flushing $(\mathrm{RR}=1.06,95 \% \mathrm{Cl}=0.43$ to $2.61, P=0.90)$, and blurred vision ( $R R=0.95,95 \% \mathrm{Cl}=0.35$ to $2.58, P=0.91$ ). Besides, rescue analgesics were required less in the antimuscarinics group than in the control group ( $R R=0.51,95 \% \mathrm{Cl}=0.32$ to $0.80, P=0.003$ ).

Conclusions: Compared with the control group, the antimuscarinics group had a significant improvement on $\mathrm{CRBD}$, the patients were well tolerated and the use rate of rescue analgesics was low.

Keywords: Antimuscarinics, Catheter related bladder discomfort, Randomized controlled trials, Prevention or treatment, Systematic review, Meta-analysis

\footnotetext{
* Correspondence: doctorzzb@163.com; 3098516949@qq.com

'Zhongbao Zhou and Yuanshan Cui contributed equally to this work as COfirst authors.

'Department of Urology, Beijing TianTan Hospital, Capital Medical University, No.119 South 4th Ring West Road, Fengtai District, Beijing 100070, China

${ }^{4}$ Department of Urology, Weifang People's Hospital, Weifang 261000, Shandong, China

Full list of author information is available at the end of the article
}

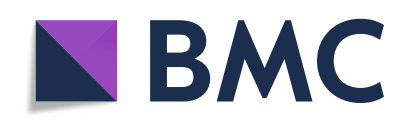

(- The Author(s). 2021 Open Access This article is licensed under a Creative Commons Attribution 4.0 International License, which permits use, sharing, adaptation, distribution and reproduction in any medium or format, as long as you give appropriate credit to the original author(s) and the source, provide a link to the Creative Commons licence, and indicate if changes were made. The images or other third party material in this article are included in the article's Creative Commons licence, unless indicated otherwise in a credit line to the material. If material is not included in the article's Creative Commons licence and your intended use is not permitted by statutory regulation or exceeds the permitted use, you will need to obtain permission directly from the copyright holder. To view a copy of this licence, visit http://creativecommons.org/licenses/by/4.0/. The Creative Commons Public Domain Dedication waiver (http://creativecommons.org/publicdomain/zero/1.0/) applies to the data made available in this article, unless otherwise stated in a credit line to the data. 


\section{Introduction}

The temporary insertion of catheter to improve intraoperative micturition and evaluation of perioperative voiding volume is essential operation for patients undergoing surgery. However, the incidence of catheter-related bladder discomfort (CRBD) immediately after operation is as high as $47 \sim 90 \%$ (Bala et al. 2012; Kunin 2001).

Patients describe CRBD as discomfort in the suprapubic area caused by indwelling a catheter or a burning sensation with micturition impulse (Binhas et al. 2011). As one of the most painful postoperative complications, CRBD increases the incidence of postoperative pain and reduces the quality of perioperative recovery (Maro et al. 2014). The exact pathophysiology of CRBD is not clear. However, its symptoms are similar to overactive bladder (frequent and urgent micturition with or without urgent urinary incontinence) caused by the involuntary contraction of bladder mediated by muscarinic receptors located in the urothelium and efferent nerve (Abrams et al. 2014; Andersson 1999). Five muscarinic receptor subtypes (M1-M5) in vivo mediate distinct physiological functions according to their locations and receptor subtypes (Bai et al. 2015). All of them have been detected in the bladder; it was thought that the most physiologically relevant subtypes were M2 and M3, with M2 being more abundant in the detrusor muscle but M3 being more active in eliciting detrusor contraction (Burden and Abrams 2016). M2 and M3 are mainly located in the detrusor muscle, urothelium, and efferent nerves (Yamanishi et al. 2001). Catheterization can stimulate the afferent nerve of bladder, leading to the release of acetylcholine, which causes muscarinic receptor-mediated involuntary contractions of detrusor muscle (Yamanishi et al. 2001).

At present, antimuscarinics have been reported to be an effective drug in preventing and treating CRBD, including tolterodine, solifenacin, oxybutynin, butyl scopolamine, and so on. But sometimes, side effects were observed. A recent review indicated that antimuscarinics seemed to achieve a better improvement in the clinical symptoms and a significant reduction in the incidence of CRBD compared with placebo, although these studies observed a high incidence of intervention-related side effects, in general, patients tolerated these treatments well (Bai et al. 2015). However, there was no comprehensive meta-analysis to assess the efficacy and safety of antimuscarinics in CRBD.

Therefore, we did a meta-analysis to evaluate the characteristic of antimuscarinics for CRBD in patients who underwent various surgical procedures where a catheter was inserted.

\section{Materials and methods Study protocol}

The Preferred Reporting Items for Systematic Reviews and Meta-Analyses (PRISMA) checklist was used to analysis of randomized controlled trials (RCTs) (Moher et al. 2010).

\section{Inclusion criteria}

RCTs met the following criteria: (1) antimuscarinics in preventing or treating CRBD were studied; (2) the study should consist of analyzable data referred to efficacy and safety, mainly including the number of patients with CRBD, dry mouth, postoperative nausea and vomiting (PONV), facial flushing, blurred vision, or rescue analgesics; and (3) the full text of articles should be available. If the above criteria were not met, the study was removed from our study.

\section{Search strategy}

We searched MEDLINE, Embase, and Cochrane Controlled Trials Register databases to identify RCTs published before July 2021 using the following key words: antimuscarinics, anticholinergic, solifenacin, darifenacin, tolterodine, oxybutynin, scopolamine, glycopyrrolate, atropine, CRBD, and RCT. We confined our search to published studies in English only. The author reviewed the references of articles as well. We also tried to contact the authors of articles that we could not view full text.

\section{Trial selection}

Three authors independently identified relevant studies according to inclusion criteria. Any discrepancies were recorded, discussed, and settled in a negotiated manner. If the identical study was published in different journals or at different time, the latest study was included in the analysis. However, if a group of patients was involved in two or more studies, each study may be included.

\section{Quality assessment}

We used the Cochrane Handbook for Systematic Reviews of Interventions 2nd Edition to assess the quality of each study (Cumpston et al. 2019). We evaluated the methodological quality according to selection bias, performance bias, detection bias, attrition bias, reporting bias, and other bias. The quality of each study was classified as one of three degrees: "+" if the study satisfied all quality criteria, the study had a low risk of bias; "?" if the study had one or more ambiguous quality criteria, the study had a moderate risk of bias; "-" if the study met few quality criteria, the study had a high risk of bias. All authors assessed the quality of RCTs and agreed with the final results. 


\section{Data extraction}

We collected the following data from each study: (1) the name of first-author and the publishing year of the article, (2) study design, (3) the method of therapy; (4) sample size, (5) catheter type, (6) timing of administration, (7) anesthesia type; (8) ASA score and (9) surgery type; (10) basic information of patients, including age, sex, weight, duration of surgery and anesthesia; and (11) number of loss to follow-up, whether to calculation of sample size, the method of statistical analysis, and whether to intention-to-treat (ITT) analysis.

\section{Statistical analysis}

The present meta-analysis was carried out by using $\mathrm{Re}$ view Manager version 5.4.0 (Cochrane Collaboration, Oxford, UK) (Cumpston et al. 2019). The results were expressed as the risk ratio (RR) for discontinuous outcomes with 95\% confidence intervals (CI) (DerSimonian and Laird 1986). We assessed the degree of heterogeneity with Cochrane's $Q$ tests and $I^{2}$ statistics. $P$ value $\leq$ 0.05 or $I^{2} \geq 50 \%$ reflected a significant heterogeneity. To reduce the heterogeneity, a random-effects model was used in the study. $P<0.05$ was considered statistically significant. Due to the insufficient number of included studies, we did not perform subgroup analysis to analyze the source of heterogeneity.

\section{Results}

Study selection process and characteristics of studies

Our search strategy found 170 articles in these databases. After reviewing their abstracts and titles, we ruled out 138 articles. Among the remaining 32 articles, 21 articles were excluded for lack of analyzable data. Finally, eleven studies (Agarwal et al. 2006; Agarwal et al. 2005; Chung et al. 2017; Kim et al. 2016; Maghsoudi et al. 2018; Nam et al. 2015; Ryu et al. 2013; Sabetian et al. 2017; Şahiner et al. 2020; Srivastava et al. 2016; TauzinFin et al. 2007) involving 1165 patients were studied in the analysis (Fig. 1). The basic characteristics of RCTs were listed in Table 1 and Table 2.

\section{Quality of individual studies}

Eleven studies included in the analysis were RCTs. All studies had an appropriate number of participants to

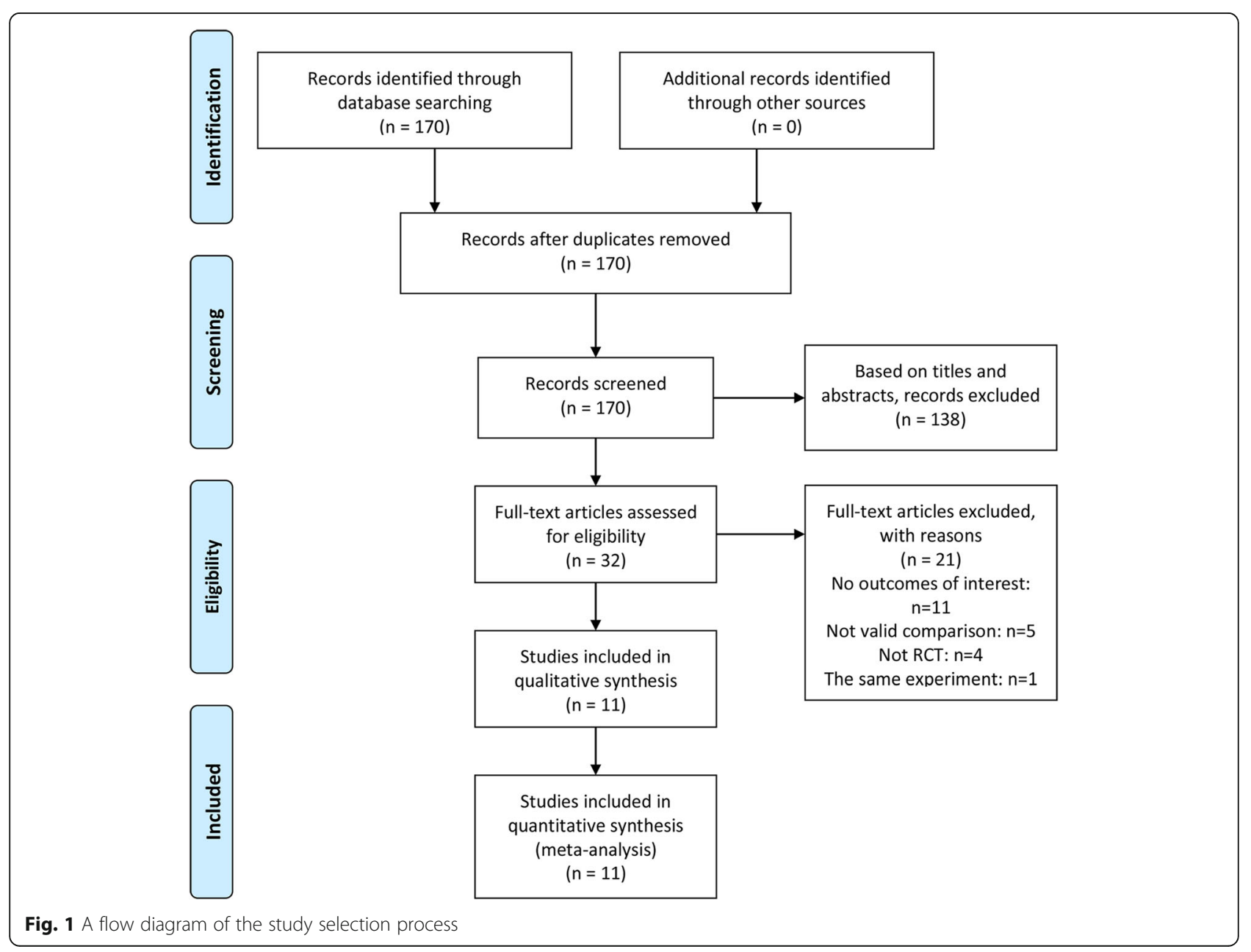


Table 1 The details of individual study

\begin{tabular}{|c|c|c|c|c|c|c|c|c|c|c|}
\hline \multirow[t]{2}{*}{ Study } & \multirow{2}{*}{$\begin{array}{l}\text { Study } \\
\text { design }\end{array}$} & \multirow{2}{*}{$\begin{array}{l}\text { Therapy in } \\
\text { experimental } \\
\text { group }\end{array}$} & \multirow{2}{*}{$\begin{array}{l}\text { Therapy } \\
\text { in } \\
\text { control } \\
\text { group }\end{array}$} & \multicolumn{2}{|l|}{ Sample size } & \multirow{2}{*}{$\begin{array}{l}\text { Catheter } \\
\text { type }\end{array}$} & \multirow{2}{*}{$\begin{array}{l}\text { Timing of } \\
\text { administration }\end{array}$} & \multirow{2}{*}{$\begin{array}{l}\text { Anesthesia } \\
\text { type }\end{array}$} & \multirow{2}{*}{$\begin{array}{l}\text { ASA } \\
\text { score }\end{array}$} & \multirow[t]{2}{*}{ Surgery type } \\
\hline & & & & Experimental & Control & & & & & \\
\hline $\begin{array}{l}\text { Agarwal } \\
\text { et al. } 2005\end{array}$ & $\mathrm{RCT}$ & $\begin{array}{l}\text { Tolterodine } 2 \\
\text { mg oral }\end{array}$ & Placebo & 50 & 165 & 16 Foley & $\begin{array}{l}1 \mathrm{~h} \text { before } \\
\text { induction of } \\
\text { anesthesia }\end{array}$ & $\begin{array}{l}\text { General } \\
\text { anesthesia }\end{array}$ & $|/| \mid$ & $\begin{array}{l}\text { Urologic surgery for } \\
\text { kidney and ureter }\end{array}$ \\
\hline $\begin{array}{l}\text { Agarwal } \\
\text { et al. } 2006\end{array}$ & $\mathrm{RCT}$ & $\begin{array}{l}\text { Oxybutynin } \\
5 \mathrm{mg} / \\
\text { Tolterodine } 2 \\
\text { mg oral }\end{array}$ & Placebo & $78 / 78$ & 78 & 16 Foley & $\begin{array}{l}1 \mathrm{~h} \text { before } \\
\text { induction of } \\
\text { anesthesia }\end{array}$ & $\begin{array}{l}\text { General } \\
\text { anesthesia }\end{array}$ & $|/| \mid$ & $\begin{array}{l}\text { Percutaneous } \\
\text { nephrolithotomy } \\
\text { surgery }\end{array}$ \\
\hline $\begin{array}{l}\text { Tauzin-Fin } \\
\text { et al. } 2007\end{array}$ & $\mathrm{RCT}$ & $\begin{array}{l}\text { Oxybutynin } 5 \\
\text { mg sublingual }\end{array}$ & Placebo & 23 & 23 & 16 Foley & $\begin{array}{l}\text { Every } 8 \mathrm{~h} \text { for the } \\
\text { first } 24 \mathrm{~h} \text { after } \\
\text { surgery }\end{array}$ & $\begin{array}{l}\text { General } \\
\text { anesthesia }\end{array}$ & $|/| \mid$ & $\begin{array}{l}\text { Radical retropubic } \\
\text { prostatectomy }\end{array}$ \\
\hline $\begin{array}{l}\text { Ryu et al. } \\
2013\end{array}$ & $\mathrm{RCT}$ & $\begin{array}{l}\text { Butyl } \\
\text { scopolamine } \\
20 \text { mg iv }\end{array}$ & Saline & 28 & 29 & $\begin{array}{l}16 / 18 \\
\text { Foley }\end{array}$ & $\begin{array}{l}\text { After reporting } \\
\text { CRBD }\end{array}$ & $\begin{array}{l}\text { General } \\
\text { anesthesia }\end{array}$ & $|/| \mid$ & $\begin{array}{l}\text { Urethrolithotomy, } \\
\text { nephrolithotomy } \\
\text { and radical } \\
\text { retropubic } \\
\text { prostatectomy }\end{array}$ \\
\hline $\begin{array}{l}\text { Nam et al. } \\
2015\end{array}$ & $\mathrm{RCT}$ & $\begin{array}{l}\text { Butyl } \\
\text { scopolamine } \\
20 \mathrm{mg} \text { iv }\end{array}$ & None & 49 & 50 & 14 Foley & $\begin{array}{l}\text { Intravenously } \\
\text { immediately } \\
\text { before the end } \\
\text { of the operation }\end{array}$ & $\begin{array}{l}\text { General } \\
\text { anesthesia }\end{array}$ & $|/| \mid$ & $\begin{array}{l}\text { Stomach, } \\
\text { hepatobiliary, } \\
\text { colorectal operation }\end{array}$ \\
\hline $\begin{array}{l}\text { Chung } \\
\text { et al. } 2017\end{array}$ & $\mathrm{RCT}$ & $\begin{array}{l}\text { Solifenacin } 5 \\
\text { mg oral }\end{array}$ & None & 62 & 72 & 18 Foley & $\begin{array}{l}\text { The day before, } \\
\text { the day of, and } \\
\text { the day after } \\
\text { surgery }\end{array}$ & $\begin{array}{l}\text { General } \\
\text { anesthesia }\end{array}$ & NA & $\begin{array}{l}\text { Transurethral } \\
\text { resection of bladder } \\
\text { tumor }\end{array}$ \\
\hline $\begin{array}{l}\text { Srivastava } \\
\text { et al. } 2016\end{array}$ & $\mathrm{RCT}$ & $\begin{array}{l}\text { Solifenacin } 5 \\
\text { mg/ } \\
\text { Darifenacin } 7.5 \\
\text { mg oral }\end{array}$ & Placebo & $30 / 30$ & 30 & 16 Foley & $\begin{array}{l}1 \mathrm{~h} \text { prior to } \\
\text { induction of } \\
\text { anesthesia }\end{array}$ & $\begin{array}{l}\text { General } \\
\text { anesthesia }\end{array}$ & $|/| \mid$ & $\begin{array}{l}\text { Elective spine } \\
\text { surgery }\end{array}$ \\
\hline $\begin{array}{l}\text { Sabetian } \\
\text { et al. } 2017\end{array}$ & $\mathrm{RCT}$ & $\begin{array}{l}\text { Hyoscine } N \text { - } \\
\text { butyl bromide } \\
20 \text { mg iv }\end{array}$ & $\begin{array}{l}\text { Saline } \\
1 \mathrm{ml} \text { iv }\end{array}$ & 24 & 26 & 22 Foley & $\begin{array}{l}\text { Intravenously } \\
\text { before the } \\
\text { induction of } \\
\text { anesthesia }\end{array}$ & $\begin{array}{l}\text { General } \\
\text { anesthesia }\end{array}$ & $|/| \mid$ & $\begin{array}{l}\text { Transurethral } \\
\text { resection of prostate }\end{array}$ \\
\hline $\begin{array}{l}\text { Maghsoudi } \\
\text { et al. } 2018\end{array}$ & $\mathrm{RCT}$ & $\begin{array}{l}\text { Tolterodine } 2 \\
\text { mg oral }\end{array}$ & $\begin{array}{l}\text { Vitamin } \\
\text { C } 250 \\
\text { mg oral }\end{array}$ & 50 & 70 & 16 Foley & $\begin{array}{l}1 \mathrm{~h} \text { before } \\
\text { surgery }\end{array}$ & $\begin{array}{l}\text { General } \\
\text { anesthesia }\end{array}$ & I & $\begin{array}{l}\text { Percutaneous } \\
\text { nephrolithotomy }\end{array}$ \\
\hline $\begin{array}{l}\text { Kim et al. } \\
2016\end{array}$ & $\mathrm{RCT}$ & $\begin{array}{l}\text { Glycopyrrolate } \\
0.3 \mathrm{mg} \text { iv }\end{array}$ & $\begin{array}{l}\text { Saline } 1.5 \\
\mathrm{ml} \text { iv }\end{array}$ & 30 & 30 & 16 Foley & $\begin{array}{l}\text { Intravenously } \\
\text { before the } \\
\text { induction of } \\
\text { anesthesia }\end{array}$ & $\begin{array}{l}\text { General } \\
\text { anesthesia }\end{array}$ & $|/| \mid$ & $\begin{array}{l}\text { Ureteroscopic } \\
\text { removal of ureter } \\
\text { stone }\end{array}$ \\
\hline $\begin{array}{l}\text { Şahiner } \\
\text { et al. } 2020\end{array}$ & $\mathrm{RCT}$ & $\begin{array}{l}\text { Atropine } 15 \\
\mu \mathrm{g} / \mathrm{kg} \text { iv }\end{array}$ & Placebo & 30 & 30 & $\begin{array}{l}16-20 \\
\text { Foley }\end{array}$ & $\begin{array}{l}\text { The end of the } \\
\text { surgery }\end{array}$ & $\begin{array}{l}\text { General } \\
\text { anesthesia }\end{array}$ & $|/||/|||$ & $\begin{array}{l}\text { Transurethral } \\
\text { resection }\end{array}$ \\
\hline
\end{tabular}

$R C T$ randomized controlled trial, ASA American Society of Anesthesiologists, NA not available, CRBD catheter related bladder discomfort

analyze, and no study showed intention-to-treat analysis (Table 2). Risk of bias summary and graph were showed in Fig. 2. The funnel plot showed a qualitative estimate of the publication bias of the study. The icons of each indicator were evenly distributed on both sides of the vertical line, which implied that no evidence of bias was found (Fig. 3).

Antimuscarinics versus placebo on the incidence of CRBD Eleven RCTs including 1059 patients (509 in the antimuscarinics group and 550 in the control group) were used to assess the impact of antimuscarinic on the incidence of CRBD.

\section{The incidence of CRBD at $0 \mathrm{~h}$}

The analysis was supplied by ten RCTs including 1002 patients, which showed heterogeneity $P$ value of $<$ 0.00001 and $I^{2}$ of $85 \%$. Forest plots drew a RR of 0.61 and $95 \% \mathrm{CI}$ of 0.46 to $0.82(P=0.001)$, which indicated that patients treated with antimuscarinics had a lower incidence of CRBD than those treated with placebo at 0h postoperatively (Fig. 4).

\section{The incidence of CRBD at $1 \mathrm{~h}$}

The analysis was supplied by ten RCTs including 1002 patients, which showed heterogeneity $P$ value of 0.0002 and $I^{2}$ of $72 \%$. Forest plots drew a RR of 0.60 and $95 \%$ 
Table $\mathbf{2}$ The characteristics of patients

\begin{tabular}{|c|c|c|c|c|c|c|c|c|c|c|c|c|c|c|}
\hline \multirow[t]{2}{*}{ Study } & \multicolumn{2}{|c|}{$\begin{array}{l}\text { Age (years) } \\
\text { Mean } \pm \text { SD } \\
\text { (Range) }\end{array}$} & \multicolumn{2}{|c|}{$\begin{array}{l}\text { Sex } \\
\text { (Male/ } \\
\text { Female) }\end{array}$} & \multicolumn{2}{|c|}{$\begin{array}{l}\text { Weight }(\mathbf{k g}) \\
\text { Mean } \pm \text { SD }\end{array}$} & \multicolumn{2}{|c|}{$\begin{array}{l}\text { Duration of } \\
\text { surgery }(\mathrm{min}) \\
\text { Mean } \pm \text { SD }\end{array}$} & \multicolumn{2}{|c|}{$\begin{array}{l}\text { Duration } \\
\text { of } \\
\text { anesthesia } \\
\text { (min) } \\
\text { Mean } \pm \text { SD }\end{array}$} & \multirow[t]{2}{*}{$\begin{array}{l}\text { Loss } \\
\text { to } \\
\text { follow- } \\
\text { up }\end{array}$} & \multirow[t]{2}{*}{$\begin{array}{l}\text { Calculation } \\
\text { of sample } \\
\text { size }\end{array}$} & \multirow[t]{2}{*}{$\begin{array}{l}\text { Statistical } \\
\text { analysis }\end{array}$} & \multirow[t]{2}{*}{$\begin{array}{l}\text { ITT } \\
\text { analysis }\end{array}$} \\
\hline & EXP & $\mathrm{CON}$ & EXP & $\mathrm{CON}$ & EXP & CON & EXP & $\mathrm{CON}$ & EXP & CON & & & & \\
\hline $\begin{array}{l}\text { Agarwal } \\
\text { et al. } 2005\end{array}$ & $\begin{array}{l}40.2 \pm \\
13.6\end{array}$ & $\begin{array}{l}42.6 \\
\pm \\
14.4\end{array}$ & $\begin{array}{l}33 / \\
17\end{array}$ & $\begin{array}{l}114 / \\
51\end{array}$ & $\begin{array}{l}56.90 \pm \\
8.09\end{array}$ & $\begin{array}{l}58.24 \pm \\
11.4\end{array}$ & NA & & NA & & 0 & Yes & $\begin{array}{l}T \text { tests; Chi- } \\
\text { square test; }\end{array}$ & No \\
\hline $\begin{array}{l}\text { Agarwal } \\
\text { et al. } 2006\end{array}$ & $\begin{array}{l}45.6 \pm \\
13.2 \\
44.4 \pm \\
12.8\end{array}$ & $\begin{array}{l}43.6 \\
\pm \\
14.4\end{array}$ & $\begin{array}{l}40 / \\
38 \\
39 / \\
39\end{array}$ & $\begin{array}{l}42 / \\
36\end{array}$ & $\begin{array}{l}55.4 \pm \\
12.6 \\
56.9 \pm \\
10.2\end{array}$ & $\begin{array}{l}57.3 \pm \\
11.4\end{array}$ & NA & & NA & & 0 & Yes & $\begin{array}{l}\text { Chi-square test; } \\
\text { ANOVA }\end{array}$ & No \\
\hline $\begin{array}{l}\text { Tauzin-Fin } \\
\text { et al. } 2007\end{array}$ & $\begin{array}{l}65.8(54- \\
72)\end{array}$ & $\begin{array}{l}61.1 \\
(54- \\
75)\end{array}$ & Male & & $\begin{array}{l}74.1 \pm \\
9.1\end{array}$ & $\begin{array}{l}78.1 \pm \\
13.8\end{array}$ & $\begin{array}{l}188.2 \pm \\
31.3\end{array}$ & $\begin{array}{l}181.8 \\
\pm \\
33.8\end{array}$ & NA & & 0 & Yes & $\begin{array}{l}T \text { tests; Chi- } \\
\text { square test }\end{array}$ & No \\
\hline $\begin{array}{l}\text { Ryu et al. } \\
2013\end{array}$ & $\begin{array}{l}61(22- \\
70)\end{array}$ & $\begin{array}{l}61 \\
(24- \\
69)\end{array}$ & Male & & $67 \pm 10$ & $70 \pm 11$ & $178 \pm 66$ & $\begin{array}{l}190 \pm \\
76\end{array}$ & $\begin{array}{l}224 \\
\pm 70\end{array}$ & $\begin{array}{l}238 \\
\pm 82\end{array}$ & 0 & Yes & $\begin{array}{l}T \text { tests; Chi- } \\
\text { square test }\end{array}$ & No \\
\hline $\begin{array}{l}\text { Nam et al. } \\
2015\end{array}$ & $58 \pm 10$ & $\begin{array}{l}60 \pm \\
9\end{array}$ & Male & & $69 \pm 11$ & $67 \pm 9$ & $154 \pm 89$ & $\begin{array}{l}160 \pm \\
95\end{array}$ & $\begin{array}{l}198 \\
\pm 95\end{array}$ & $\begin{array}{l}203 \\
\pm \\
101\end{array}$ & 0 & Yes & $\begin{array}{l}\text { Chi-square test; } \\
\text { ANOVA }\end{array}$ & No \\
\hline $\begin{array}{l}\text { Chung } \\
\text { et al. } 2017\end{array}$ & $\begin{array}{l}66(43- \\
84)\end{array}$ & $\begin{array}{l}68 \\
(26- \\
85)\end{array}$ & $\begin{array}{l}51 / \\
11\end{array}$ & $\begin{array}{l}61 / \\
11\end{array}$ & NA & & NA & & NA & & 0 & Yes & ANOVA; $T$ tests & No \\
\hline $\begin{array}{l}\text { Srivastava } \\
\text { et al. } 2016\end{array}$ & $\begin{array}{l}46.8 \pm \\
9.6 ; 43.0 \\
\pm 7.9\end{array}$ & $\begin{array}{l}48.2 \\
\pm \\
10.2\end{array}$ & $\begin{array}{l}24 / \\
6 ; \\
26 / \\
4\end{array}$ & $25 / 5$ & $\begin{array}{l}63.1 \pm \\
8.6 ; 65.0 \\
\pm 10.3\end{array}$ & $\begin{array}{l}61.1 \pm \\
9.5\end{array}$ & $\begin{array}{l}149.8 \pm \\
28.5 \\
161.5 \pm \\
40.5\end{array}$ & $\begin{array}{l}156.7 \\
\pm \\
35.8\end{array}$ & NA & & 0 & Yes & ANOVA & No \\
\hline $\begin{array}{l}\text { Sabetian } \\
\text { et al. } 2017\end{array}$ & $\begin{array}{l}64.95 \pm \\
7.88\end{array}$ & $\begin{array}{l}63.34 \\
\pm \\
9.22\end{array}$ & Male & & $\begin{array}{l}66.29 \pm \\
6.77\end{array}$ & $\begin{array}{l}65.61 \pm \\
7.21\end{array}$ & $\begin{array}{l}73.12 \pm \\
20.42\end{array}$ & $\begin{array}{l}76.34 \\
\pm \\
23.64\end{array}$ & NA & & 0 & Yes & $\begin{array}{l}T \text { tests; Chi- } \\
\text { square test; } \\
\text { ANOVA }\end{array}$ & No \\
\hline $\begin{array}{l}\text { Maghsoudi } \\
\text { et al. } 2018\end{array}$ & $\begin{array}{l}44.4 \pm \\
9.7\end{array}$ & $\begin{array}{l}44.1 \\
\pm \\
12.2\end{array}$ & NA & & $\begin{array}{l}25.9 \pm \\
3.1(\mathrm{BMl}, \\
\left.\mathrm{kg} / \mathrm{m}^{2}\right)\end{array}$ & $\begin{array}{l}25.0 \pm \\
4.2(\mathrm{BMl} \\
\left.\mathrm{kg} / \mathrm{m}^{2}\right)\end{array}$ & $\begin{array}{l}97.9 \pm \\
19.7\end{array}$ & $\begin{array}{l}105.9 \\
\pm \\
23.4\end{array}$ & NA & & 0 & Yes & $T$ tests; ANOVA & No \\
\hline $\begin{array}{l}\text { Kim et al. } \\
2016\end{array}$ & $\begin{array}{l}48.6 \pm \\
12.2\end{array}$ & $\begin{array}{l}50.2 \\
\pm \\
14.5\end{array}$ & $\begin{array}{l}29 / \\
11\end{array}$ & $\begin{array}{l}33 / \\
10\end{array}$ & $\begin{array}{l}70.4 \pm \\
12.8\end{array}$ & $\begin{array}{l}70.2 \pm \\
11.0\end{array}$ & $\begin{array}{l}18.7 \pm \\
12.2\end{array}$ & $\begin{array}{l}23.0 \\
\pm \\
15.9\end{array}$ & $\begin{array}{l}37.9 \\
\pm \\
13.1\end{array}$ & $\begin{array}{l}39.3 \\
\pm \\
19.1\end{array}$ & 0 & Yes & $\begin{array}{l}\text { Chi-square test; } \\
\text { Fisher's exact } \\
\text { test; } T \text { tests }\end{array}$ & No \\
\hline $\begin{array}{l}\text { Şahiner } \\
\text { et al. } 2020\end{array}$ & $\begin{array}{l}59.7 \pm \\
2.8\end{array}$ & $\begin{array}{l}58.7 \\
\pm 2.5\end{array}$ & $\begin{array}{l}25 / \\
5\end{array}$ & $25 / 5$ & $\begin{array}{l}27.9 \pm \\
3.9(\mathrm{BMl} \\
\left.\mathrm{kg} / \mathrm{m}^{2}\right)\end{array}$ & $\begin{array}{l}27.2 \pm \\
3.4(\mathrm{BMl} \\
\left.\mathrm{kg} / \mathrm{m}^{2}\right)\end{array}$ & $\begin{array}{l}41.5 \pm \\
18.8\end{array}$ & $\begin{array}{l}30.7 \\
\pm \\
14.6\end{array}$ & $\begin{array}{l}53.3 \\
\pm \\
21.5\end{array}$ & $\begin{array}{l}38.0 \\
\pm \\
17.8\end{array}$ & 0 & Yes & $\begin{array}{l}\text { Mann-Whitney } \\
\text { U-test; T-tests; } \\
\text { Chi-square test; }\end{array}$ & No \\
\hline
\end{tabular}

EXP experimental group, CON control group, ITT intention-to-treat, ANOVA analysis of variance, SD standard deviation, NA not available

CI of 0.47 to $0.76(P<0.0001)$, which indicated that patients treated with antimuscarinics had a statistical reduction of CRBD than those treated with placebo at 1-h postoperatively (Fig. 4).

\section{The incidence of CRBD at $2 \mathrm{~h}$}

The analysis was supplied by eight RCTs including 822 patients, which showed heterogeneity $P$ value of 0.002 and $I^{2}$ of $68 \%$. Forest plots drew a RR of 0.58 and $95 \%$ $\mathrm{CI}$ of 0.43 to $0.79(P=0.0005)$, which indicated that patients treated with antimuscarinics had a statistical reduction of CRBD than those treated with placebo at 2-h postoperatively (Fig. 4).

\section{The incidence of CRBD at $6 \mathrm{~h}$}

The analysis was supplied by eight RCTs including 755 patients, which showed heterogeneity $P$ value of < 0.00001 and $I^{2}$ of $80 \%$. Forest plots drew a RR of 0.48 and $95 \% \mathrm{CI}$ of 0.31 to $0.75(P=0.001)$, which indicated that patients treated with antimuscarinics had a statistical reduction of CRBD than those treated with placebo at 6-h postoperatively (Fig. 4).

\section{Antimuscarinics versus placebo on the incidence of adverse event}

The adverse event including dry mouth, PONV, facial flushing, and blurred vision was reported in the included RCTs. The number of patients that needed rescue 


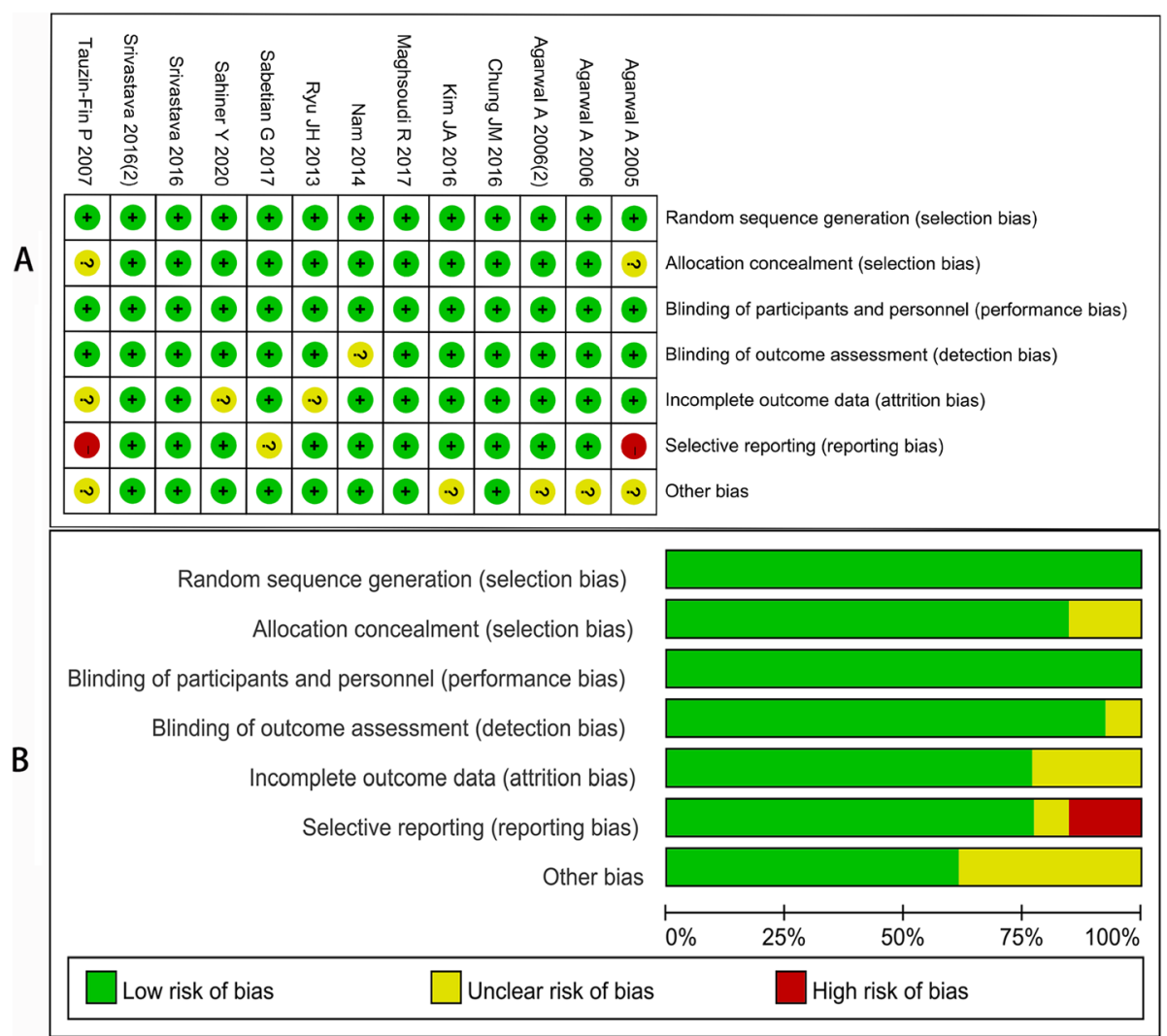

Fig. 2 Risk of bias summary (A) and graph (B)

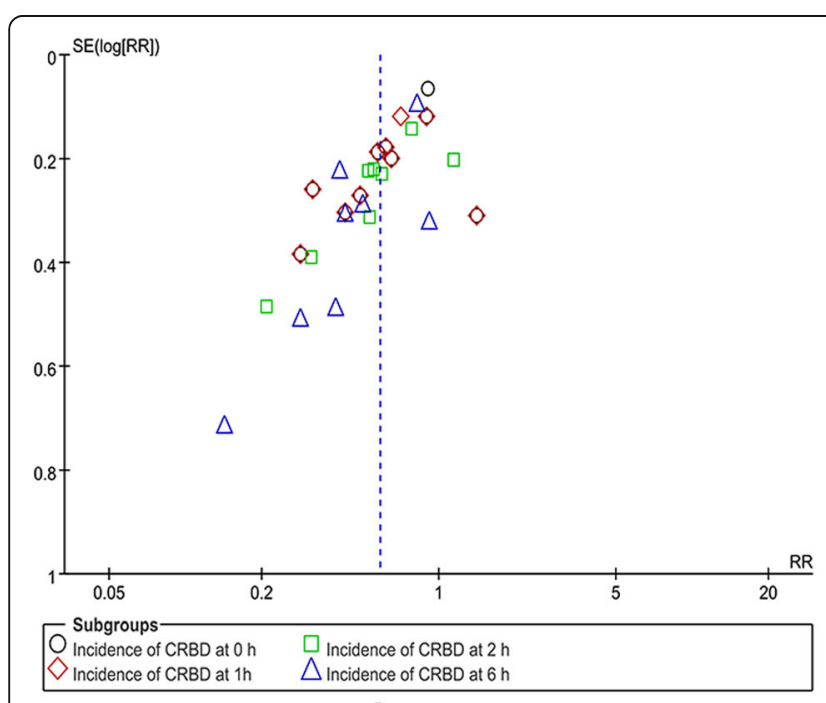

A

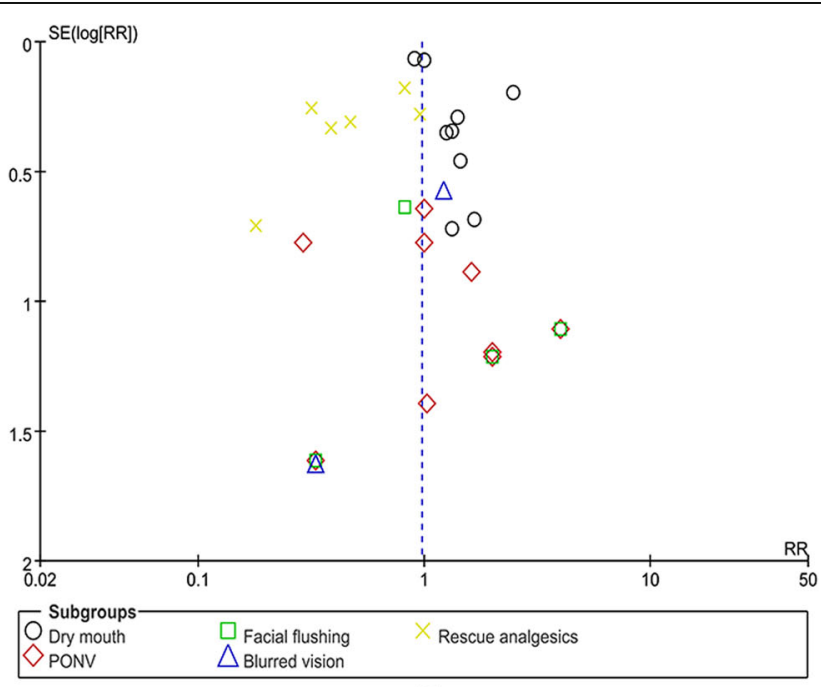

B

Fig. 3 Funnel plot of the studies included in our meta-analysis. RR risk ratio, PONV postoperative nausea and vomiting 


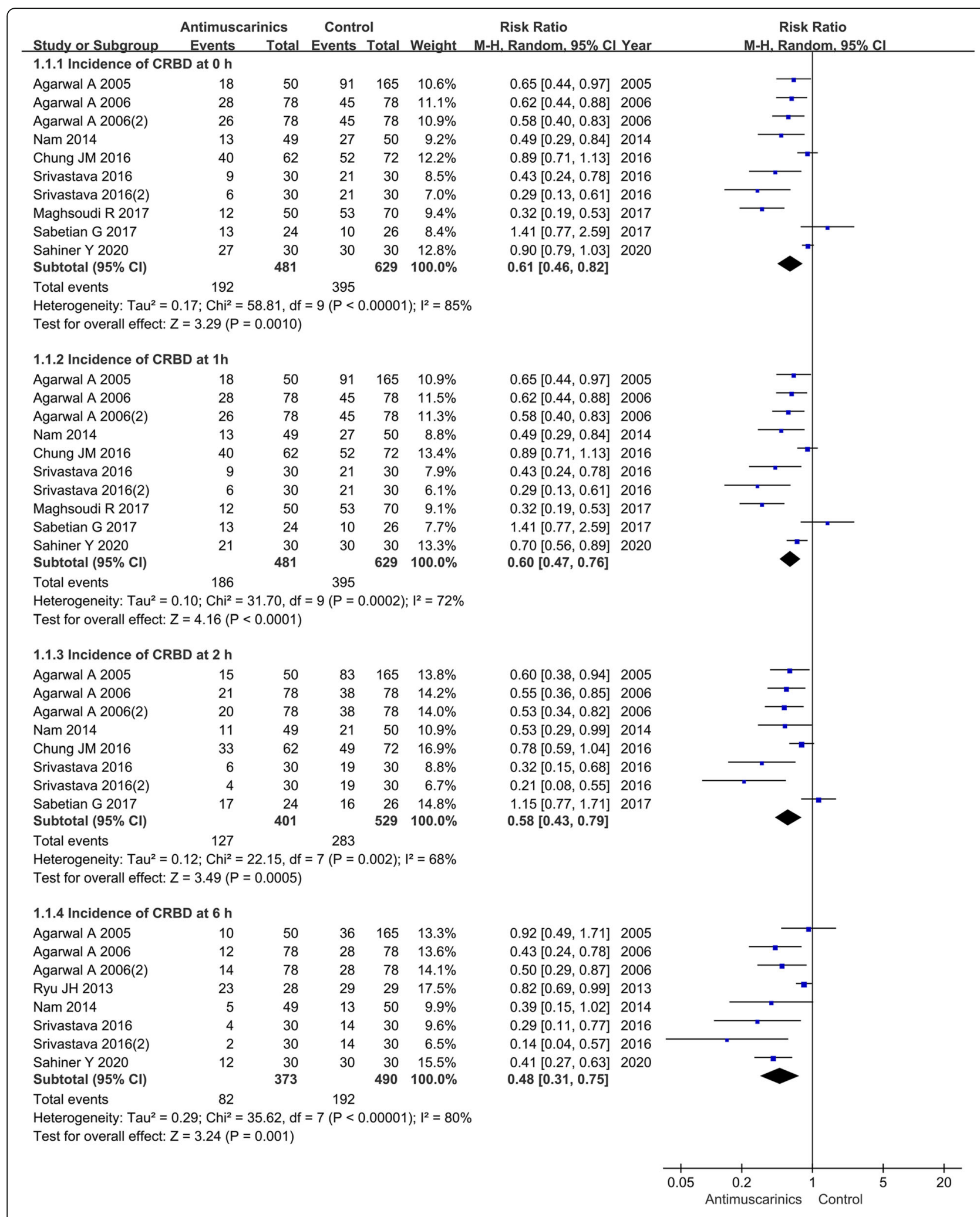

Fig. 4 The incidence of CRBD in antimuscarinics versus placebo. $M-H$ Mantel-Haenszel, Cl confidence interval, $d f$ degrees of freedom, $R R$ risk ratio 
analgesics postoperatively in two groups was analyzed in our study. The results showed that there was no difference in the incidence of adverse effects between the antimuscarinics and control groups. Rescue analgesics were required less for patients in the antimuscarinics group than those in the control group (Fig. 5).

\section{Dry mouth}

Ten RCTs involving 888 patients reported the occurrence of dry mouth, which showed heterogeneity $P$ value of $<0.00001$ and $I^{2}$ of $83 \%$. The result indicated that antimuscarinics were not significantly different from placebo in the incidence of dry mouth (RR 1.31, 95\% CI 0.95-1.80, $P=0.09$ ).

\section{PONV}

Nine RCTs involving 669 patients reported the occurrence of PONV, which showed heterogeneity $P$ value of 0.70 and $I^{2}$ of $0 \%$. The result showed that there was no statistical difference between the two groups in the incidence of PONV (RR 1.02, 95\% CI 0.55-1.90, $P=0.94$ ).

\section{Facial flushing}

Six RCTs involving 483 patients reported the occurrence of facial flushing, which showed heterogeneity $P$ value of 0.57 and $I^{2}$ of $0 \%$. The result showed that there was no statistical difference between the two groups in the incidence of facial flushing (RR 1.06, 95\% CI 0.43-2.61, $P=$ 0.90).

\section{Blurred vision}

Four RCTs involving 393 patients reported the occurrence of blurred vision, which showed heterogeneity $P$ value of 0.59 and $I^{2}$ of $0 \%$. The result showed that there was no statistical difference between the two groups in the incidence of blurred vision (RR 0.95, 95\% CI 0.352.58, $P=0.91)$.

\section{Rescue analgesics}

Seven RCTs involving 527 patients reported the number of rescue analgesics, which showed heterogeneity $P$ value of 0.003 and $I^{2}$ of $72 \%$. The result showed that the number of patients who needed rescue analgesics postoperatively was less in the antimuscarinics group compared with the control group (RR $0.51,95 \%$ CI $0.32-0.80, P=$ 0.003).

\section{Discussion}

Clinically, patients with indwelling urinary catheter were reported to suffer from a variety of catheter-related symptoms, such as suprapubic pain, frequency, urgency, and urge incontinence (Agarwal et al. 2005; Akça et al. 2016). Further, patients may have behavioral responses to CRBD, including agitation, loud complaints, and attempt to remove the bladder catheter accompanied by a burning sensation in the urethra, which resulted in the increased incidence of postoperative complications (Binhas et al. 2011).

Antimuscarinics have been widely used to treat overactive bladder for many years (White and Iglesia 2016). Because the symptoms of CRBD and overactive bladder $(\mathrm{OAB})$ were similar, various muscarinic antagonists have been used in the treatment of CRBD. Antimuscarinics can block the muscarinic receptors of detrusor and prevent the stimulation of neurotransmitter acetylcholine. Thereby, they can reduce the frequency and intensity of detrusor contraction. Moreover, antimuscarinics have been proved to inhibit bladder-afferent mechanisms and increase the capacity of bladder (Dimitropoulos and Gravas 2015). Based on these pathways, antimuscarinics can effectively prevent or alleviate CRBD. In our study, it was also found that antimuscarinics statistically decreased the incidence of CRBD. However, antimuscarinics not only function in the bladder, but also in other parts of the body with muscarinic receptors which can result in some side effects, such as dry mouth, PONV, facial flushing, etc. (Abrams and Andersson 2007).

This meta-analysis identified that the incidence of CRBD had a statistically significant reduction at $0-, 1-$, 2-, and 6-h after surgery for patient treated with antimuscarinics $(P=0.001, P<0.0002, P=0.0005$, and $P=$ 0.001 , respectively). Safety assessment indicated that there were no statistical differences between antimuscarinics and placebo for side effects including dry mouth $(P=0.09)$, PONV $(P=0.94)$, facial flushing $(P=0.90)$, and blurred vision $(P=0.91)$. Besides, the number of patients who needed rescue analgesics postoperatively was less in the antimuscarinics group compared with the control group $(P=0.003)$. After a systematic analysis of the results, the study suggested the superiority of antimuscarinics in overcoming CRBD compared with placebo.

A better understanding of the pathophysiology of CRBD was the key to better management and reduction of incidence (Bai et al. 2015). Based on this concept, a variety of treatment options have been implemented, such as the insertion of catheter can cause detrusor contraction and the activity of inflammatory mediators, thereby promoting the synthesis of prostaglandins (PG) (Andersson 2010). Therefore, paracetamol, as a PG synthesis inhibitor, may improve the incidence and symptoms of CRBD (Ergenoglu et al. 2012). In addition, doctors should consider the occurrence of side effects when deciding which drug to use. For example, antimuscarinics (e.g., solifenacin and oxybutynin) are oral drugs that can cause adverse effects including dry mouth, facial flushing, and blurred vision. Therefore, it is necessary to evaluate its dose-response titration of each drug and the 


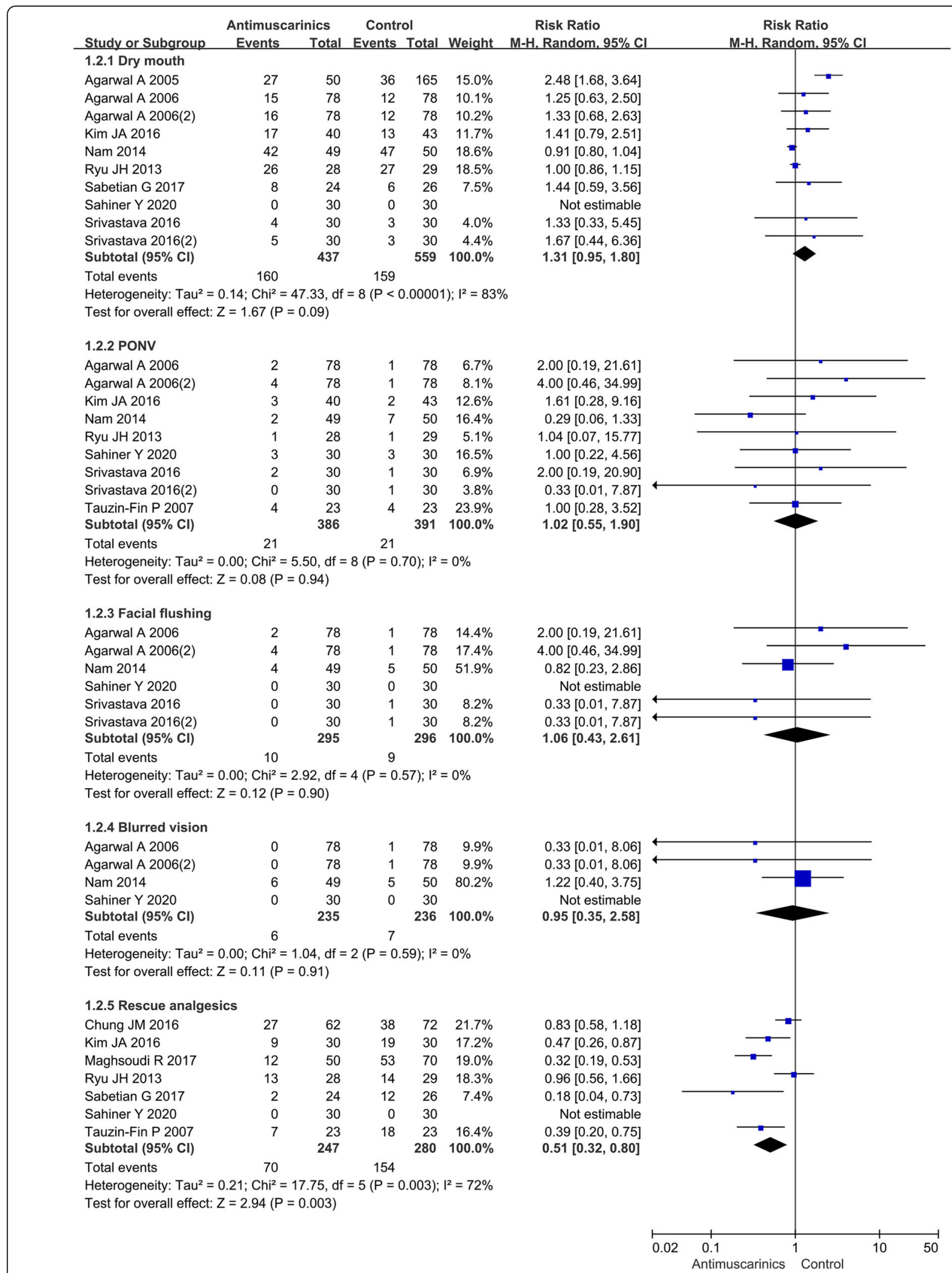

Fig. 5 The incidence of adverse events in antimuscarinics versus placebo. $M-H$ Mantel-Haenszel, $C l$ confidence interval, $d f$ degrees of freedom, $R R$ risk ratio 
effect of treating CRBD (Bai et al. 2015). Simultaneously, it is noteworthy that preoperative periurethral local anesthesia infiltration can reduce immediate postoperative pain, but this method also needs to be further evaluated to determine its effect and safety (Kumar et al. 2013; Tommaselli et al. 2014).

The studies included in this analysis were all RCTs, which increased the strength of the results. Although all included studies were of high quality, our study also had some limitations. Firstly, this meta-analysis only included 11 studies with small sample size, which was limited to the number of relevant original studies. Secondly, the patient's perioperative indicators may be inconsistent, including different sizes of catheter, different kinds of medications, different doses of medications, different timing of administration, and different ASA score. Thirdly, the patients included in the analysis have undergone various types of surgery. Among them, patients undergoing transurethral surgery had a higher incidence of CRBD, which may have an impact on the results. Finally, this paper involved the treatment or prevention of CRBD, but we cannot conduct a subgroup-analysis due to the small number of included articles, which may lead to different results. Therefore, high-quality RCTs were needed to explore the efficacy and safety of antimuscarinics for CRBD.

\section{Conclusions}

Compared with the control group, the antimuscarinics group had a significant improvement on CRBD, the patients were well tolerated, and the use rate of rescue analgesics was low.

\section{Abbreviations \\ CRBD: Catheter related bladder discomfort; PRISMA: Preferred Reporting Items for Systematic Reviews and Meta-Analyses; RCTs: Randomized controlled trials; RR: Risk ratio; Cl: Confidence interval; PONV: Postoperative nausea and vomiting; OAB: Overactive bladder}

\section{Acknowledgements}

The authors would like to thank the authors of the primary studies.

\section{Authors' contributions}

All authors provided substantial contributions to the conception and design. $Z Z, Y C$, and $X Z$ conducted data collection. ZZ, YC, YL, and ZC performed statistical analysis. ZZ and $Y C$ drafted the original manuscript. $Y Z$ and $Z C$ reviewed and revised the article. All authors read and approved the final manuscript.

\section{Funding}

This work was supported by the National Nature Science Foundation of China (81801429), Shandong Provincial Natural Science Foundation, China (ZR2017LH016), and Yantai Science and Technology Bureau (2018SFGY117).

\section{Availability of data and materials}

All data generated or analyzed during this study are included in this article.

\section{Declarations}

Ethics approval and consent to participate Not applicable.
Consent for publication

Not applicable.

\section{Competing interests}

The authors declare that they have no competing interests.

\section{Author details}

${ }^{1}$ Department of Urology, Beijing TianTan Hospital, Capital Medical University, No.119 South 4th Ring West Road, Fengtai District, Beijing 100070, China. ${ }^{2}$ Department of Urology, The Affiliated Yantai Yuhuangding Hospital of Qingdao University, Yantai 264000, Shandong, China. ${ }^{3}$ Department of Urology, PLA Rocket Force Characteristic Medical Center, No. 16 Xinwai Street, Xicheng District, Beijing 100088, China. ${ }^{4}$ Department of Urology, Weifang People's Hospital, Weifang 261000, Shandong, China.

Received: 26 September 2020 Accepted: 6 September 2021

Published online: 14 December 2021

\section{References}

Abrams P, Andersson KE. Muscarinic receptor antagonists for overactive bladder. BJU Int. 2007;100(5):987-1006. https://doi.org/10.1111/j.1464-410X.2007.07205. $\mathrm{x}$.

Abrams P, Cardozo L, Wein A. Fourth international consultation on incontinenceresearch society 2013. Neurourol Urodyn. 2014;33(5):571-2. https://doi.org/1 0.1002/nau.22617.

Agarwal A, Dhiraaj S, Singhal V, Kapoor R, Tandon M. Comparison of efficacy of oxybutynin and tolterodine for prevention of catheter related bladder discomfort: a prospective, randomized, placebo-controlled, double-blind study. Br J Anaesth. 2006;96(3):377-80. https://doi.org/10.1093/bja/ael003.

Agarwal A, Raza M, Singhal V, Dhiraaj S, Kapoor R, Srivastava A, et al. The efficacy of tolterodine for prevention of catheter-related bladder discomfort: a prospective, randomized, placebo-controlled, double-blind study. Anesth Analg. 2005;101(4):1065-7, table of contents. https://doi.org/10.1213/01.ane. 0000167775.46192.e9.

Akça, B., Aydoğan-Eren, E., Canbay, Ö., Karagöz, A. H., Üzümcügil, F., Ankay-Yilbaş, A., \& Celebi, N. (2016). Comparison of efficacy of prophylactic ketamine and dexmedetomidine on postoperative bladder catheter-related discomfort. Saudi Med J, 37(1), 55-59. doi:https://doi.org/10.15537/smj.2016.1.14122

Andersson KE. Advances in the pharmacological control of the bladder. Exp Physiol. 1999;84(1):195-213. https://doi.org/10.1111/j.1469-445x.1999. tb00083.x.

Andersson KE. Detrusor myocyte activity and afferent signaling. Neurourol Urodyn. 2010;29(1):97-106. https://doi.org/10.1002/nau.20784.

Bai Y, Wang X, Li X, Pu C, Yuan H, Tang Y, et al. Management of Catheter-Related Bladder Discomfort in Patients Who Underwent Elective Surgery. J Endourol. 2015;29(6):640-9. https://doi.org/10.1089/end.2014.0670.

Bala I, Bharti N, Chaubey VK, Mandal AK. Efficacy of gabapentin for prevention of postoperative catheter-related bladder discomfort in patients undergoing transurethral resection of bladder tumor. Urology. 2012;79(4):853-7. https:// doi.org/10.1016/j.urology.2011.11.050.

Binhas M, Motamed C, Hawajri N, Yiou R, Marty J. Predictors of catheter-related bladder discomfort in the post-anaesthesia care unit. Ann Fr Anesth Reanim. 2011;30(2):122-5. https://doi.org/10.1016/j.annfar.2010.12.009.

Burden $\mathrm{H}$, Abrams P. Urinary incontinence in men: current and developing therapy options. Expert Opin Pharmacother. 2016;17(5):715-26. https://doi. org/10.1517/14656566.2016.1145662.

Chung JM, Ha HK, Kim DH, Joo J, Kim S, Sohn DW, et al. Evaluation of the efficacy of solifenacin for preventing catheter-related bladder discomfort after transurethral resection of bladder tumors in patients with non-muscle invasive bladder cancer: a prospective, randomized, multicenter study. Clin Genitourin Cancer. 2017;15(1):157-62. https://doi.org/10.1016/j.clgc.2016.05. 006.

Cumpston M, Li T, Page MJ, Chandler J, Welch VA, Higgins JP, et al. Updated guidance for trusted systematic reviews: a new edition of the Cochrane Handbook for Systematic Reviews of Interventions. Cochrane Database Syst Rev. 2019;10:Ed000142. https://doi.org/10.1002/14651858.Ed000142.

DerSimonian R, Laird N. Meta-analysis in clinical trials. Control Clin Trials. 1986; 7(3):177-88. https://doi.org/10.1016/0197-2456(86)90046-2.

Dimitropoulos K, Gravas S. Solifenacin/tamsulosin fixed-dose combination therapy to treat lower urinary tract symptoms in patients with benign 
prostatic hyperplasia. Drug Des Devel Ther. 2015;9:1707-16. https://doi.org/1 $0.2147 /$ dddt.S53184.

Ergenoglu P, Akin S, Yalcin Cok O, Eker E, Kuzgunbay B, Turunc T, et al. Effect of intraoperative paracetamol on catheter-related bladder discomfort: a prospective, randomized, double-blind study. Curr Ther Res Clin Exp. 2012; 73(6):186-94. https://doi.org/10.1016/j.curtheres.2012.08.001.

Kim JA, Min JH, Lee HS, Jo HR, Je UJ, Paek JH. Effects of glycopyrrolate premedication on preventing postoperative catheter-related bladder discomfort in patients receiving ureteroscopic removal of ureter stone. Korean J Anesthesiol. 2016;69(6):563-7. https://doi.org/10.4097/kjae.2016.69.6. 563.

Kumar A, Griwan MS, Singh SK, Sen J, Pawar DS. Is periprostatic nerve block a gold standard in case of transrectal ultrasound-guided prostate biopsy? Urol Ann. 2013;5(3):152-6. https://doi.org/10.4103/0974-7796.115732.

Kunin CM. Nosocomial urinary tract infections and the indwelling catheter: what is new and what is true? Chest. 2001;120(1):10-2. https://doi.org/10.1378/ chest.120.1.10.

Maghsoudi R, Farhadi-Niaki S, Etemadian M, Kashi AH, Shadpour P, Shirani A, et al. Comparing the efficacy of tolterodine and gabapentin versus placebo in catheter related bladder discomfort after percutaneous nephrolithotomy: a randomized clinical trial. J Endourol. 2018;32(2):168-74. https://doi.org/10.1 089/end.2017.0563.

Maro S, Zarattin D, Baron T, Bourez S, de la Taille A, Salomon L. Catheter-related bladder discomfort after urological surgery: importance of the type of surgery and efficiency of treatment by clonazepam. Prog Urol. 2014;24(10): 628-33. https://doi.org/10.1016/j.purol.2014.05.002.

Moher D, Liberati A, Tetzlaff J, Altman DG. Preferred reporting items for systematic reviews and meta-analyses: the PRISMA statement. Int J Surg. 2010;8(5):336-41. https://doi.org/10.1016/j.jiju.2010.02.007.

Nam K, Seo JH, Ryu JH, Oh AY, Lee T, Park HP, et al. Randomized, clinical trial on the preventive effects of butylscopolamine on early postoperative catheterrelated bladder discomfort. Surgery. 2015;157(2):396-401. https://doi.org/10.1 016/j.surg.2014.05.017

Ryu JH, Hwang JW, Lee JW, Seo JH, Park HP, Oh AY, et al. Efficacy of butylscopolamine for the treatment of catheter-related bladder discomfort: a prospective, randomized, placebo-controlled, double-blind study. Br J Anaesth. 2013;111(6):932-7. https://doi.org/10.1093/bja/aet249.

Sabetian G, Zand F, Asadpour E, Ghorbani M, Adibi P, Hosseini MM, et al. Evaluation of hyoscine N-butyl bromide efficacy on the prevention of catheter-related bladder discomfort after transurethral resection of prostate: a randomized, double-blind control trial. Int Urol Nephrol. 2017;49(11):1907-13. https://doi.org/10.1007/s11255-017-1663-2.

Şahiner Y, Yağan Ö, Akdağıı Ekici A, Ekici M, Demir E. The effect of atropine in preventing catheter-related pain and discomfort in patients undergoing transurethral resection due to bladder tumor; prospective randomized, controlled study. Korean J Pain. 2020;33(2):176-82. https://doi.org/10.3344/ kjp.2020.33.2.176.

Srivastava VK, Nigam R, Agrawal S, Kumar S, Rambhad S, Kanaskar J. Evaluation of the efficacy of solifenacin and darifenacin for prevention of catheter-related bladder discomfort: a prospective, randomized, placebo-controlled, doubleblind study. Minerva Anestesiol. 2016;82(8):867-73.

Tauzin-Fin P, Sesay M, Svartz L, Krol-Houdek MC, Maurette P. Sublingual oxybutynin reduces postoperative pain related to indwelling bladder catheter after radical retropubic prostatectomy. Br J Anaesth. 2007:99(4):5725. https://doi.org/10.1093/bja/aem232.

Tommaselli GA, Di Carlo C, Formisano C, Fabozzi A, Nappi C. Effect of local infiltration analgesia on post-operative pain following TVT-O: a double-blind, placebo-controlled randomized study. Arch Gynecol Obstet. 2014;290(2):2839. https://doi.org/10.1007/s00404-014-3186-8.

White N, Iglesia CB. Overactive bladder. Obstet Gynecol Clin North Am. 2016; 43(1):59-68. https://doi.org/10.1016/j.ogc.2015.10.002.

Yamanishi T, Chapple CR, Chess-Williams R. Which muscarinic receptor is important in the bladder? World J Urol. 2001;19(5):299-306. https://doi.org/1 $0.1007 / 5003450100226$.

\section{Publisher's Note}

Springer Nature remains neutral with regard to jurisdictional claims in published maps and institutional affiliations.

\section{Ready to submit your research? Choose BMC and benefit from:}

- fast, convenient online submission

- thorough peer review by experienced researchers in your field

- rapid publication on acceptance

- support for research data, including large and complex data types

- gold Open Access which fosters wider collaboration and increased citations

- maximum visibility for your research: over $100 \mathrm{M}$ website views per year

At BMC, research is always in progress.

Learn more biomedcentral.com/submissions 